\title{
Statins and oxidative stress in the cardiovascular system
}

\author{
Marios Margaritis*, Fabio Sanna*, Charalambos Antoniades
}

Division of Cardiovascular Medicine, Radcliffe Department of Medicine, University of Oxford United Kingdom

Word count: 5,081

Figures: 1

*: equal author contribution

\section{Corresponding author:}

Charalambos Antoniades MD PhD

Division of Cardiovascular Medicine, Radcliffe Department of Medicine,

University of Oxford, West Wing L6 John Radcliffe Hospital, Headley Way

Oxford OX3 9DU, United Kingdom

Tel: +44-1865-221870

Fax: +44-1865-740352

e-mail: Charalambos.antoniades@cardiov.ox.ac.uk 


\begin{abstract}
Statins are widely established as an important class of medications for primary and secondary prevention of cardiovascular disease. In addition to their lipid-lowering effects, mounting evidence suggests that statins exhibit non-lipid-lowering mediated effects in the cardiovascular system. These so called "pleiotropic" effects are partly due to antioxidant properties of statins. These are mediated by inhibition of the mevalonate pathway, which interferes with small GTP-ase protein prenylation. This, in turn, leads to anti-oxidant effects of statins via a plethora of mechanisms. Statins prevent the activation of the pro-oxidant enzyme NADPH-oxidase by interfering with Rac1 activation and translocation to the membrane, as well as reducing expression of crucial subunits of NADPH-oxidase. Statins also enhancing the expression, enzymatic activity and coupling of endothelial nitric oxide synthase (eNOS), through mevalonate-dependent effects. The net result is a restoration of the redox balance in the cardiovascular system, with subsequent anti-atherosclerotic and cardioprotective effects. While the evidence from basic science studies and animal models is strong, more clinical trials are required to establish the relevance of these pleiotropic effects to human cardiovascular disease and potentially lead to expanded indications for statin treatment or alternative therapeutic strategies.
\end{abstract}


Primary and secondary prevention of cardiovascular disease (CVD) is at the cornerstone of everyday medical practice in cardiology. Statins form one of the most important weapons in our therapeutic arsenal. Large randomised clinical trials have established without a doubt the pivotal position that statins play in reducing cardiovascular risk. For example, they have been shown to lower incidence of events in patients with established low(1) or high risk of $\operatorname{CVD}(2)$, as a primary preventative approach. In secondary prevention, they have been shown to protect against development of post-procedural infarction of the myocardium(3) and to reduce all-cause mortality in patients suffering from an acute coronary syndrome (ACS) (4), as well as non-fatal events in stable and acute coronary patients (5).

Most of these effects are achieved through the primary mechanism of actions of statins, which is reduction of cholesterol synthesis and reduction of circulating low density lipoprotein cholesterol (LDL-C) levels. Some studies, however, have demonstrated beneficial lipid-lowering independent effects of statins, which have been termed pleiotropic. These are mostly mediated through the anti-inflammatory and anti-oxidant effects of statins. This review focuses on the role of statins as an antioxidant intervention targeting the cardiovascular system.

\section{Sources of free radicals in the vascular wall}

Reactive oxygen species (ROS) are unstable oxygen-containing moieties with a very short half-life. They react readily with proteins, lipids and DNA and lead to disruption of these biological molecules. ROS production in the cardiovascular system originates from multiple enzymatic sources, the most important of which are NADPH-oxidases(6) and uncoupled endothelial nitric oxide synthase (eNOS). Other sources of ROS include the 
inducible form of nitric oxide synthase (iNOS) as well as the uncoupled eNOS, xanthine oxidase, mitochondrial oxidases and other enzymatic systems (7).

\section{NADPH-oxidases}

The multi-subunit, membrane enzyme NADPH-oxidase was first identified in phagocytes, where they play a crucial role in host defense by producing bacteriocidal $\mathrm{O}_{2}{ }^{\cdot-}(8)$. Since then, several distinct NADPH-oxidases have been described in various cells, based on the presence of different homologues of the catalytic membrane subunit (NOX1, NOX2 (gp91phox), NOX3, NOX4, NOX5, DUOX1 and DUOX2). The phagocytic NADPH-oxidase comprises two membrane-bound subunits: p22 $2^{\text {phox }}$ and NOX2 (constituting the cytochrome b558 complex) and four cytosolic subunits: p40 phox $, \mathrm{p} 47^{\text {phox }}, \mathrm{p} 67^{\text {phox }}$ and the guaninenucleotide-binding protein Rac1. After stimulation, Rac1 assembles the membrane subunits into a complex which translocates to the membrane and activates the enzyme. Homologues of cytosolic subunits have been identified: NoxO1 is a homologue of $\mathrm{p} 47^{\mathrm{phox}}$ and NoxA1 shares considerable similarity with $\mathrm{p} 67^{\text {phox }}(9)$. Human vascular cells express NOX1, NOX2 (which is the homologue of phagocyte NADPH-oxidase), NOX4 and NOX5(10). However, this expression profile varies considerably in different cells of the vascular wall, as well as in different vessels. Endothelial cells (ECs) express NOX1, NOX2, NOX4 and NOX5, with NOX2 being the most abundant isoform in human vein endothelium(11). In VSMCs, NOX4 is the most prevalent isoform, located primarily in the arterial media, although NOX1 and NOX2 are also present(8). Our group has shown that human cardiomyocytes express NOX2, NOX4 and very low levels of NOX5(12).

In contrast to the phagocytic NADPH-oxidase, the vascular isoforms are constitutively active, generating a low but steady amount of $\mathrm{O}_{2} \cdot{ }^{--}$, the production rate of which is significantly lower than that in phagocytes(10). This rate can be increased by various stimuli, 
such as angiotensin II, tumor necrosis factor-a (TNF-a), vascular endothelial growth factor (VEGF) or mechanical forces like shear stress. The constitutive and rather low activity of vascular NADPH-oxidases would seem to imply that under physiological conditions ROS generated by these enzymes could act as a second messenger in physiological vascular redox signaling. In disease states however, elevated expression and activity of NADPH-oxidases, resulting in elevated ROS generation, contribute to the initiation and maintenance of a plethora of cardiovascular disease states. Indeed, elevated activity of NADPH-oxidases has been specifically implicated in the development of hypertension(13), atherosclerosis(14), atrial fibrillation(15), post-operative atrial fibrillation(16), heart failure(17), as well as in the vascular complications of type 2 diabetes(18).

\section{Endothelial nitric oxide synthase}

Endothelial nitric oxide synthase (eNOS) is a dimeric membrane enzyme located predominantly in ECs, but also in VSMCs, cardiomyocytes and platelets. eNOS is a homodimer which catalyzes the conversion of L-arginine and molecular oxygen $\left(\mathrm{O}_{2}\right)$ into $\mathrm{NO}$ and L-citrulline. This is achieved through electron transfer from the flavins of the reductase domain on one monodimer to the oxidase domain of the other monodimer, which contains an iron-binding heme active site(8). The presence of calmodulin, which is activated by calciumbinding, increases the rate of electron flow. At the heme site, the electrons are utilized to reduce and activate $\mathrm{O}_{2}$, which is in turn used to produce NO through a two-step oxidation of L-arginine. This process requires the binding of the essential co-factor tetrahydrobiopterin $\left(\mathrm{BH}_{4}\right)$. When BH4 is bound to the enzyme, eNOS is "coupled"(19). Coupling is crucial for proper function of the enzyme in human cells. Oxidative destruction of of BH4 by ROS, especially peroxynitrite (ONOO-*), leads to "uncoupling" of the enzyme: without $\mathrm{BH} 4$, the flow of electrons within eNOS is disturbed dissociating the ferrous-dioxygen complex, 
causing eNOS to convert $\mathrm{O}_{2}$ into $\mathrm{O}_{2} \cdot{ }^{-}$radicals instead of $\mathrm{NO}(20) \cdot \mathrm{O}_{2} \cdot{ }^{--}$reacts with $\mathrm{NO}$ to form $\mathrm{ONOO}^{-}$, which can further oxidize $\mathrm{BH} 4$, creating a vicious circle of further uncoupling of the enzyme. The ensuing reduction in NO generation diminishes endothelium-dependent vasorelaxation, alters vascular homeostasis and results in endothelial dysfunction(19). ONOO-- can even lead to cellular apoptosis and necrosis at high concentrations, further promoting endothelial dysfunction(21).

\section{Oxidative stress in the pathogenesis of cardiovascular disease}

ROS feature as a hallmark in our current models of early stages of the atherosclerotic process(22). Typical risk factors, such as hypertension, smoking and type 2 diabetes mellitus, have all been linked to elevated production of ROS by the vascular wall (22). Oxidation of lipoprotein molecules in the subendothelial space, primarily LDL by ROS leads to formation of oxidized LDL (oxLDL) (23). Binding of oxLDL to the lectin-like oxidized receptor (LOX) activates redox-sensitive, proinflammatory transcriptional pathways (such as nuclear factor kappa B (NF-kB)) in ECs(24). As a consequence, release of inflammatory cytokines attracts monocytes to the endothelium, and an increase in the expression of cellular adhesion molecules allows them to adhere to and infiltrate the subendothelial space. In this space, they uptake oxLDL molecules and acquire macrophage-like characteristics, leading to their transformation into foam cells. This creates a vicious cycle where a heightened inflammatory state leads to more monocytes being attracted to the subendothelial space, while at the same time VSMCs migrated to the intimal layer of the vessel from the medial layer. The combination of these two processes forms the atherosclerotic plaque.

In addition to atherosclerosis, mounting evidence suggests that overproduction/reduced elimination of ROS is implicated in the pathogenesis of a plethora of cardiovascular disease states. In atrial fibrillation, ROS are involved in the pathophysiological processes leading to 
structural and electrical remodelling of the myocardium, involving multiple enzymatic systems in the process(25). We have previously shown that NADPH-oxidases activity and ONOO-- generation in the human myocardium are independent predictors of post-operative atrial fibrillation after cardiac surgery(16).

Based on the important role of ROS in the pathogenesis of atherosclerosis and myocardial disease, targeting the enzymatic systems responsible for their overproduction appears to be a rational therapeutic strategy in primary and secondary prevention of CVD.

\section{Evaluating oxidative stress in cardiovascular disease}

There are several techniques that can be implemented to determine oxidative stress in cardiovascular disease, which we have reviewed previously(26). Some are used to directly measure ROS production, with each technique having its merits and drawbacks. For example, chemiluminescence-based assays, such as lucigenin-enhanced chemiluminescence, rely on the reaction of free radicals with a luminescent probe, which produces photons that can be measured by a luminometer. Dihydroethidium staining (DHE) with or without high performance liquid chromatography (HPLC) relies on the reaction of DHE with $\mathrm{O}_{2} \cdot^{-}$to generate 2-hydroxy-ethidium, which is thought to be a specific product. Electron spin resonance (ESR) allows direct in vivo detection of ROS but is expensive and difficult to perform.

Because ROS are molecules with extremely short half-life, several assays are employed to measure more stable oxidative stress by-products as an indirect measurement of oxidative stress. Such by-products include malonyldialdehyde (MDA), 4-hydroxy-nonenal (4-HNE), isoprostanes $(\mathrm{LOOH})$ and 8-hydroxy-deoxyguanosine $(8-\mathrm{OH}-\mathrm{dG})$. The techniques used to quantify them range from simple chemical reactions with a colourimetric readout, such as the 
MDA-thiobarbituric acid assay, to enzyme-linked immunosorbent assays (ELISA), HPLC and mass spectrometry.

The vast majority of techniques used to estimate oxidative stress are used primarily for research purposes and do not form part of the routine clinical diagnostic arsenal.

\section{Pleiotropic effects of statins}

Statins act by inhibiting hydroxyl-methyl-glutaryl coenzyme A reductase, which is the rate-limiting enzyme in cholesterol synthesis in the liver. HMG-CoA reductase acts by synthesizing L-mevalonate from HMG-CoA. Mevalonate is subsequently converted, in a multi-step process, to squalene, the precursor of cholesterol. Inhibition of this process eventually results in reduced synthesis of cholesterol and subsequently LDL-C.

However, mevalonate is also a precursor to the isoprenoids farnesylpyrophosphate (FPP), and geranyl-geranyl-pyrophosphate (GGPP). These molecules can be attached to proteins, in a post-translation process called prenylation, and alter their function. Typical targets of protein prenylation are the family of small G-proteins Rho, Rac and Ras(27). Farnesylation or geranylgeranylation is crucial for activation of Rac and Rho(28). Geranylgeranylation of Rho leads to activation of the protein Rho kinase (ROCK); the Rho/ROCK pathway displays the ability to control a variety of cellular functions, such as redox signalling, proliferation/migration and apoptosis(29). More crucially, as described previously, Rac is a necessary subunit of the NOX1- and NOX2-containing NADPH-oxidase isoforms and plays a pivotal role in activation of their respective enzymatic complex, thus leading to increased production of $\mathrm{O}_{2}{ }^{-}$.

The mevalonate pathway is a biochemical component of hepatocytes, where cholesterol synthesis takes place, but is also active in cells of the cardiovascular system. Hydrophilic statins such as rosuvastatin are uptaken by hepatocytes via active transport. On the contrary, 
lipophilic statins (such as simvastatin or atorvastatin) passively diffuse through the cellular membrane and can enter diverse cell types in the human body(30). By inhibiting the prenylation of small GTPases in cells of the cardiovascular system, statins display rapid pleiotropic effects which are independent of their lipid-lowering effects.

The early statin trials examined subjects with high levels of LDL-C and focused on the lipid lowering effects of statins. Further analysis of the LIPID trial showed a reduced incidence of new-onset coronary events or need for repeat revascularization in patients with established CVD, which was independent of their LDL-C levels (31). Post-hoc analysis of the POST-CABG trial showed that intensive statin treatment conferred improved saphenous veins graft patency after CABG independently of lipid-lowering(32). The pleiotropic effects of statins were established by analysis of the JUPITER trial, in which healthy subjects were treated with rosuvastatin and exhibited reduced levels of C-reactive protein (CRP), independently of any effect on LDL-C levels(33). These studies provided proof for the existence of lipid-lowering independent effects of statins in the cardiovascular system.

\section{Antioxidant effects of statins in the cardiovascular system}

Statins \& the vascular wall

Several cell culture studies have investigated the effects of statins on the vascular wall through their ability to suppress NADPH-oxidases activity. VSMCs treated with cerivastatin exhibit enhanced membrane translocation of Rac1 leading to activation of NADPH-oxidase complexes and increase in $\mathrm{O}_{2} \cdot{ }^{--}$production after withdrawal of the statin from the incubation medium(34). NADPH-oxidases can also exert their effects through formation of lipid membrane rafts on the surface of the cell; in human coronary artery endothelial cells, statin treatment inhibits oxLDL-induced formation of lipid membrane rafts, thus leading to a reduction in $\mathrm{O}_{2} \cdot{ }^{--}$production(35). In addition to reducing NADPH-oxidases activity, statins also reduce expression of NADPH-oxidases subunits. VSMCs treated with atorvastatin 
exhibit reduced expression of NOX1 and subsequently reduced membrane translocation of Rac1, leading to reduced generation of $\mathrm{O}_{2} \cdot{ }^{-}(36)$. Statins have been shown to down-regulate $\mathrm{p} 47^{\text {phox }}$ and $\mathrm{p} 22^{\text {phox }}$ mRNA protein levels by increasing PPARa activity(37). Similar findings are applicable to porcine coronary arteries, where statin treatment reduces p22phox mRNA levels, a crucial membrane subunit of NOX1, NOX2 and NOX4 isoforms of the NADPHoxidase enzyme(38).

The results from cell cultures studies have been replicated in vivo via the use of animal models. Statin treatment reduces NADPH-oxidases activity through mevalonate-dependent inhibition of Rac activation and membrane translocation, thus improving vascular NO availability and overall endothelial function(39). In a rabbit atherosclerotic model, treatment with fluvastatin protects against high-fat diet-induced $\mathrm{p} 22^{\text {phox }}$ and NOX2 upregulation, thus ameliorating endothelial function and even leading to a reduction in atherosclerotic plaque burden(40). In a similar manner, simvastatin treatment reduces ROS generation and restores coronary artery endothelial function in a diabetic rat model(41). In hypertensive rats with normal cholesterol levels, statins have been shown to down-regulate $\mathrm{p} 22^{\text {phox }}$ and NOX1 gene expression(36), in addition to $\mathrm{p} 22^{\text {phox }}$ and angiotensin receptor protein levels, improving endothelial function via reduced ROS production(42). In an ischemic stroke rat model, atorvastatin prevents elevated NADPH-oxidases activity caused by ischaemia by reducing

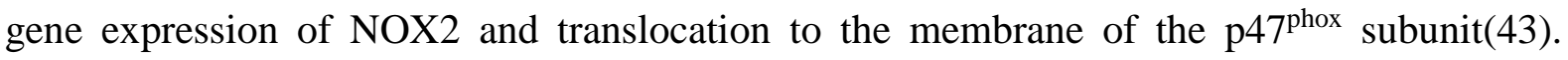
Similarly, withdrawing statin treatment in mice leads to endothelial dysfunction, through increased ROS production by Rac1-mediated activation of NADPH-oxidases(44).

Statins also exert their beneficial pleiotropic effects in the vasculature through enhanced eNOS expression, on a transcriptional and post-transcriptional level(45). Geranylgeranylation of the small GTP-ase Rho is linked to down-regulation of eNOS expression in endothelial cells. Treatment with statins inhibits GGPP formation via the mechanisms mentioned 
previously and as such leads to elevated eNOS expression(46). Moreover, increased levels of LDL have been linked to reduced eNOS mRNA expression in human umbilical vein endothelial cells, an effect reversed by simvastatin(47). This is due to protective effects of statins on eNOS mRNA stability; statins inhibit Rho, leading to changes in the actin cytoskeleton which result in elevated eNOS mRNA polyadenylation(48). Treatment of senescent endothelial cells with statins enhances eNOS expression via activation of the PI3K/Akt pathway(49). The increased expression of eNOS mediated by statins translates into improved endothelial function, as evidenced in animal models of age-related (50) and highfat diet-induced (51) endothelial dysfunction.

Statins lead to enhanced eNOS activity at a post-translational level as well. Treatment of endothelial cells with fluvastatin increases phosphorylation of eNOS at the activation sites Ser-1177 and Ser-633 through PI3K/Akt- and PKA-mediated pathways respectively(52). In endothelial cells, the enhanced eNOS phosphorylation at Ser-1177 caused by statins is partly due to increased heat shock protein-90 (hsp90) recruitment of Akt to the eNOS complex(53). Atorvastatin increases eNOS phosphorylation at Ser-633 in endothelial cells through AMPKmediated pathways as well(54). A similar mechanism has been demonstrated after ex vivo incubation of rat mesenteric resistance arteries where simvastatin leads to enhanced AMPKmediated eNOS phosphorylation at Ser-1177(55). Moreover, statins increase eNOS activity by interfering with caveolae, which are invaginations of the cellular membrane, the formation of which relies on the protein caveolin-1, which binds eNOS and leads to its inhibition. In endothelial cells, treatment with atorvastatin reduces caveolin-1 content and activates eNOS independently of LDL-C presence in the incubation medium(56). This mechanism has been replicated in vivo by using ApoE-knockout mice, where treatment with rosuvastatin reduces caveolin-1 content (57). Of course, it should be noted that activation of eNOS does not always lead to increased NO production; if eNOS is uncoupled, it would lead to increased 
production of $\mathrm{O}_{2} \cdot-$ instead of $\mathrm{NO}(58)$. However, statins enhance eNOS coupling, by increasing levels of $\mathrm{BH} 4$ : exposure of endothelial cells to statins leads to up-regulation of GTP-cyclohydrolase (GTPCH) expression -the rate-limiting enzyme of BH4 synthesis- with a subsequent increase in BH4 bioavailability, leading to enhanced eNOS coupling $(52,59)$. Similarly, in a diabetic animal model, atorvastatin administration prevents eNOS uncoupling via the same pathway(60).

The results from the cell culture and animal models have been shown to be applicable to human physiology as well. Treatment with statins reduces expression of NOX2 mRNA in internal mammary arteries obtained from subjects undergoing $\mathrm{CABG}(61)$. Hyperlipidaemic individuals who are treated with statins exhibit reduced circulating levels of gp91 ${ }^{\text {phox }}$ and other markers of systemic oxidative stress, suggesting a global beneficial antioxidant effect of statins(62). In a small randomized trial, 49 elective CABG patients received either placebo, irbesartan (150 mg/day), pravastatin (40 mg/day) or both for 28 days(63). The group who received statin or combination of statin and angiotensin receptor blocker displayed significantly higher eNOS and reduced NOX2 expression in the left internal mammary artery, independently of LDL-lowering(63). Our group has conducted a randomized, double-blind placebo control study of 3-day preoperative atorvastatin treatment (40 mg/day) before scheduled CABG surgery. We demonstrated that short-term pre-operative treatment with atorvastatin in patients undergoing cardiac surgery leads to a rapid reduction in $\mathrm{O}_{2} .^{-}$ generation and NADPH-oxidases activity in saphenous vein grafts, an effect which is not related to the LDL-lowering effects of statins(64). Markers of systemic oxidative stress, such as plasma malonyldialdehyde are also reduced by this short-term statin treatment. What is more, ex vivo exposure of these vessels to atorvastatin leads to reduction in $\mathrm{p}^{\text {phox }}$ and Rac1 translocation to the membrane, in a mevalonate reversible manner(64). In additional analysis of this study, we found that preoperative treatment with atorvastatin enhances eNOS coupling 
in the human internal mammary artery through increased $\mathrm{BH} 4$ content, compared to placebo(65). This leads to improved endothelial function, as evidenced in vivo by flowmediated dilatation of the brachial artery. Again, these effects were found to be independent of the LDL-lowering actions of atorvastatin. By performing ex vivo incubation of human IMAs to atorvastatin, we found an up-regulation of the $G C H 1$ gene which encodes for GTPCH. This leads to a subsequent elevation of vascular BH4 and total biopterin levels, effects which are prevented by exposure to mevalonate(65).

A schematic representation of the pleiotropic anti-oxidant effects of statins in the vascular wall is shown in Figure 1.

\section{Statins \& myocardium}

Oxidative stress plays an important role in the pathogenesis of diseases of the myocardium. High levels of atrial $\mathrm{O}_{2} \cdot{ }^{-}$production have been shown to have important roles in the ionic remodelling which occurs in atrial fibrillation. Myocardial hypertrophy, as well as heart failure correlates with increased oxidative stress(66). In human atrial myocardium, NOX2 has been proved to be the main source of $\mathrm{O}_{2}{ }^{--}$production. Moreover, the same authors observed increased NADPH-oxidases-dependent $\mathrm{O}_{2}{ }^{--}$production in patients with a history of atrial fibrillation(15). In the failing myocardium of patients with ischemic and dilated cardiomyopathy, increased Rac1 GTP-ase activity is associated with enhanced NADPH oxidases-related ROS production(67).

As stated before, statins modulate NADPH-oxidase activity by inhibiting Rac1 translocation and enhance eNOS activity. In human myocardial tissue, 4 weeks treatment with atorvastatin or pravastatin (40 mg/day) inhibit Rac1 activity and NADPH-oxidasesdependent oxidative stress(67). In a swine model of metabolic syndrome, low dose (1.5 $\mathrm{mg} / \mathrm{Kg}$ ) of atorvastatin for 14 days improved angiogenic response and increased expression 
of pro-angiogenic proteins including eNOS, phospho-eNOS and vascular endothelial growth factor (VEGF). However, the low-dose treatment with atorvastatin failed to prevent myocardial and peripheral oxidative stress(68). In a canine model simvastatin maintained the ability of coronary vessels to produce NO during heart failure and prevented the downregulation of eNOS which is normally observed during induced heart failure(69). In a rat model of chronic heart failure, atorvastatin improved cardiac function by reducing Rac1/p47 $7^{\text {hox }} / \mathrm{p} 67^{\text {phox }}$-mediated ROS generation(70).

Our group has demonstrated through a randomized, double-blind trial that short-term preCABG atorvastatin treatment leads to a significant reduction in NADPH-oxidases activity and $\mathrm{ONOO}^{-}$generation in the human myocardium(16). In a series of ex vivo experiments, we showed that the antioxidant effects of atorvastatin in human myocardial tissue were linked to its ability to suppress the mevalonate pathway, because exogenous addition of mevalonate reversed these effects(16). Given the crucial role of ROS overproduction in post-operative atrial fibrillation, clinical trials have attempted to explore the role of pre-operative short-term statin treatment in preventing post-operative atrial fibrillation after cardiac surgery. However, a recent large randomized clinical trial of almost 2000 patients receiving perioperative rosuvastatin failed to find any significant protective effect of statin treatment in patients undergoing elective surgery and was actually linked to increased risk of acute kidney injury(71). It is important to note that rosuvastatin is a largely hydrophilic statin, and would not be expected to display a significant diffusion into cardiomyocytes, which lack the active mechanisms of statin transport that hepatocytes possess, as mentioned previously. The results from this study highlight the need for more large randomized trial to assess the effects of other, more lipophilic statins, in preventing post-operative atrial fibrillation. 


\section{Statins \& platelets}

Platelets play a pivotal role in the process of atherogenesis; dysfunctional platelet activation is commonly observed in atherosclerosis(72). In physiological conditions platelets do not adhere to intact endothelium. One of the mechanism through which platelet adhesion is prevented is by eNOS activity and NO production by endothelial cells and platelets themselves $(73,74)$. As already discussed, statins inhibit prenylation of small GTP-ase, Rac1 and Rho and it has been demonstrated that geranylation of Rho leads to down-regulation of eNOS expression in endothelial cells(75). In an animal model, $1.25 \mathrm{mg} / \mathrm{Kg}$ of atorvastatin were administrated to Sprague Dawley rats for 10 days in order to assess the antithrombotic effects of statin. The authors demonstrated that in rats fed with atorvastatin eNOS protein expression was enhanced $75 \%$ compared to the control group(76). Yemisci et al. demonstrated that $(40 \mathrm{mg} / \mathrm{Kg})$ of perivastatin daily administrated for 2 weeks improves eNOS activity but not increased the expression of eNOS in human platelets(77). In a different study the effect of simvastatin $(20 \mathrm{mg} /$ day $)$, atorvastatin $(10 \mathrm{mg} /$ day $)$, fluvastatin (40 mg/day) and pravastatin $(40 \mathrm{mg} /$ day) was tested on platelets function in hypercholesterolemic subjects. Simvastatin, atorvastatin, fluvastatin and pravastatin reduce platelet activity after 1, 2, 3 and 4 weeks of treatment, respectively(78). These results suggest that the different nature of the statins used as well as different tissue permeability and metabolism could lead to different results in terms of preventing oxidative stress. 


\section{Statins as a "targeted" antioxidant paradigm}

Given that ROS play a crucial role in the pathogenesis of atherosclerosis, a large number of randomized trials having been performed, exploring the use of dietary supplements with antioxidant vitamins as a strategy for CVD prevention. However, the latest meta-analysis, comprising almost 300,000 participants enrolled in 50 different randomized trials showed that there was no evidence for a beneficial effect of these type of antioxidant supplements for the prevention $\operatorname{CVD}(79)$. The results of these trials do not disprove the role of oxidative stress in the pathogenesis of cardiovascular disease. Dietary antioxidant supplements act as indiscriminate scavengers of ROS and do not have the capacity to target enzymatic generation of free radicals. Compartmentalisation of ROS formation is a crucial feature of redox state control, as a large number of enzymatic systems generate intracellular $\operatorname{ROS}(80)$. What is more, ROS are important second messengers, mediators in several signalling transduction pathways which regulate responses to injury, hypoxia etc(81). Untargeted scavenging of ROS, which is achieved through dietary antioxidants such as vitamin $\mathrm{C}$ and $\mathrm{E}$, would both interfere with these pathways, but also be unable to specifically target those cellular compartments where excessive production of ROS is harmful. On the contrary, lipophilic statins form a paradigm for a successful "targeted" antioxidant treatment strategy in the cardiovascular system. Through their ability to penetrate the cellular membrane, they are able to reach the intracellular space; through their ability to interfere with NADPH-oxidases activation and to enhance eNOS coupling and activity, they are able to target specifically those enzymatic systems, the dysregulation of which is implicated in the development of cardiovascular disease(45). 


\section{Other lipid-lowering agents: any evidence for pleiotropic effects?}

While statins currently form the cornerstone of all lipid-lowering strategies in everyday clinical practice, several other lipid-lowering agents exist that can be used as an adjuvant, in individuals who do not tolerate statin therapy, or in patients suffering from familial hypercholesterolaemia syndromes. These agents include fibrates, ezetimibe and the novel Proprotein Convertase Subtilisin/Kexin type 9 (PCSK9) monoclonal antibodies. While their lipid-lowering abilities have been proven beyond doubt, the question remains whether these agents possess any "pleiotropic" effects that could be compared to the well-documented lipidlowering independent beneficial effects of statins. Ezetimibe, already more than 15 years on the market, has been the subject of a number of basic and clinical studies investigating the presence of pleiotropic effects. The results have been mixed, with some animal studies showing substantial atheroprotective effects(82), but most clinical studies failing to show any effect superior to statins or even any effect at all(83).

Monoclonal antibodies targeting PCSK9 have been postulated to possess additional lipid-lowering "pleiotropic" effects independent of their ability to reduce LDL cholesterol, such as possibly reducing triglyceride-rich lipoprotein production(84). In addition, independently of LDL-lowering, PCSK9 is hypothesized to potentially interfere with vascular inflammation: VSMCs generate increased PCSK9 compared to ECs in conditions of increased inflammation(84). In addition, low shear stress regions increase production of PCSK9 in VSMCs and ECs, in tandem with increased ROS generation, which seems to regulate PCSK9 expression(85). These interactions between ROS and PCSK9 could play an important role in the pathogenesis of atherosclerosis. However, a recent meta-analysis of

more than 2,500 participants did not reveal any significant effect of PCSK9 monoclonal antibody therapy on circulating levels of high sensitivity C-reactive protein(86). Large-scale 
studies are needed to establish the presence of any lipid-lowering independent effect of these novel agents.

\section{Conclusions - Future perspectives}

Statins are one of our key therapeutic approaches in our attempts to reduce cardiovascular risk both in primary and secondary prevention. Their role in cardiovascular risk prevention remains pivotal, even now following the introduction in clinical practice of novel lipid-lowering agents, such as proprotein convertase subtilisin/kexin type 9 (PCSK9) monoclonal antibodies. In addition to their beneficial lipid-lowering capabilities, statins display direct pleiotropic effects on vascular and myocardial function, suppressing ROS generation through the mechanisms reviewed in this article. Statins contribute towards restoration of the physiological redox state, both in the vascular wall and the human myocardium. They suppress the activity of NADPH-oxidases, promote increased expression, activity and coupling of eNOS and enhance the effectiveness of endogenous antioxidant defense systems. Through these mechanisms, they interfere with the ROS-dependent pathophysiological processes implicated in the development of atherosclerosis, hypertension, atrial fibrillation and a plethora of other disease-states of the cardiovascular system.

\section{Figure Legends}

Figure 1. Effects of statins in cells of the vascular wall

Statins act by inhibiting hydroxyl-methyl-glutaryl-Coenzyme A (HGM-CoA) reductase. This leads to reduce formation of isoprenoids, such as farnesyl- and geranyl-pyrophosphate, with subsequent reduced prenylation of the small GTP-ase Rac. This leads to reduced activation of NOX1 and NOX2 NADPH-oxidase isoforms. At the same time, statins reduce gene expression of NADPH-oxidase subunits NOX1, NOX2, p47 $7^{\text {phox }}, \mathrm{p} 22^{\text {phox }}$. The net effect is suppression of NADPH-oxidases activity and reduced superoxide $\left(\mathrm{O}_{2} \cdot^{-}\right)$generation from this 
enzymatic complex. Inhibition of Rho prenylation leads to enhanced endothelial nitric oxide synthase (eNOS) mRNA stability, increasing expression of the enzyme. Acting through AMP-kinase, phosphatidyl-inositol-3-kinase (PI3K)/Akt and protein kinase A (PKA)mediated pathways, statins increased phosphorylation of eNOS at the activation sites Ser1177 and Ser633, thus leading to increased activity of the enzyme. Concurrently, statins increase gene expression of $G C H 1$, which encodes for GTP-cyclohydrolase (GTPCH), thus increasing levels of tetrahydrobiopterin (BH4). This, in turn, improves coupling of eNOS. The combined effect is a net increase in nitric oxide (NO) bioavailability.

\section{References}

1. Mihaylova B, Emberson J, Blackwell L, Keech A, Simes J, Barnes EH, et al. The effects of lowering LDL cholesterol with statin therapy in people at low risk of vascular disease: meta-analysis of individual data from 27 randomised trials. Lancet. 2012;380(9841):581-90.

2. Mills EJ, O'Regan C, Eyawo O, Wu P, Mills F, Berwanger O, et al. Intensive statin therapy compared with moderate dosing for prevention of cardiovascular events: a meta-analysis of $>40000$ patients. Eur Heart J. 2011;32(11):1409-15.

3. Winchester DE, Wen X, Xie L, Bavry AA. Evidence of pre-procedural statin therapy a metaanalysis of randomized trials. Journal of the American College of Cardiology. 2010;56(14):1099-109.

4. Cannon CP, Steinberg BA, Murphy SA, Mega JL, Braunwald E. Meta-analysis of cardiovascular outcomes trials comparing intensive versus moderate statin therapy. Journal of the American College of Cardiology. 2006;48(3):438-45.

5. Afilalo J, Majdan AA, Eisenberg MJ. Intensive statin therapy in acute coronary syndromes and stable coronary heart disease: a comparative meta-analysis of randomised controlled trials. Heart. 2007;93(8):914-21.

6. Guzik TJ, West NE, Black E, McDonald D, Ratnatunga C, Pillai R, et al. Vascular superoxide production by NAD(P)H oxidase: association with endothelial dysfunction and clinical risk factors. Circulation research. 2000;86(9):E85-90.

7. Wassmann S, Wassmann K, Nickenig G. Regulation of antioxidant and oxidant enzymes in vascular cells and implications for vascular disease. Curr Hypertens Rep. 2006;8(1):69-78.

8. Channon KM, Guzik TJ. Mechanisms of superoxide production in human blood vessels: relationship to endothelial dysfunction, clinical and genetic risk factors. Journal of physiology and pharmacology : an official journal of the Polish Physiological Society. 2002;53(4 Pt 1):515-24.

9. Banfi B, Clark RA, Steger K, Krause KH. Two novel proteins activate superoxide generation by the NADPH oxidase NOX1. The Journal of biological chemistry. 2003;278(6):3510-3.

10. Lassegue B, Clempus RE. Vascular NAD(P)H oxidases: specific features, expression, and regulation. American journal of physiology Regulatory, integrative and comparative physiology. 2003;285(2):R277-97.

11. Guzik TJ, Sadowski J, Kapelak B, Jopek A, Rudzinski P, Pillai R, et al. Systemic regulation of vascular $\mathrm{NAD}(\mathrm{P}) \mathrm{H}$ oxidase activity and nox isoform expression in human arteries and veins.

Arteriosclerosis, thrombosis, and vascular biology. 2004;24(9):1614-20. 
12. Antonopoulos AS, Margaritis M, Verheule S, Recalde A, Sanna F, Herdman L, et al. Mutual Regulation of Epicardial Adipose Tissue and Myocardial Redox State by PPAR-gamma/Adiponectin Signalling. Circ Res. 2016;118(5):842-55.

13. Konior A, Schramm A, Czesnikiewicz-Guzik M, Guzik TJ. NADPH oxidases in vascular pathology. Antioxid Redox Signal. 2014;20(17):2794-814.

14. Guzik TJ, Sadowski J, Guzik B, Jopek A, Kapelak B, Przybylowski P, et al. Coronary artery superoxide production and nox isoform expression in human coronary artery disease. Arteriosclerosis, thrombosis, and vascular biology. 2006;26(2):333-9.

15. Kim YM, Guzik TJ, Zhang YH, Zhang MH, Kattach H, Ratnatunga C, et al. A myocardial Nox2 containing $\mathrm{NAD}(\mathrm{P}) \mathrm{H}$ oxidase contributes to oxidative stress in human atrial fibrillation. Circ Res. 2005;97(7):629-36.

16. Antoniades C, Demosthenous M, Reilly S, Margaritis M, Zhang MH, Antonopoulos A, et al. Myocardial redox state predicts in-hospital clinical outcome after cardiac surgery effects of shortterm pre-operative statin treatment. Journal of the American College of Cardiology. 2012;59(1):6070.

17. Munzel T, Gori T, Keaney JF, Jr., Maack C, Daiber A. Pathophysiological role of oxidative stress in systolic and diastolic heart failure and its therapeutic implications. European heart journal. 2015;36(38):2555-64.

18. Antonopoulos AS, Margaritis M, Coutinho P, Shirodaria C, Psarros C, Herdman L, et al. Adiponectin as a link between type 2 diabetes and vascular NADPH oxidase activity in the human arterial wall: the regulatory role of perivascular adipose tissue. Diabetes. 2015;64(6):2207-19.

19. Landmesser U, Hornig B, Drexler $\mathrm{H}$. Endothelial function: a critical determinant in atherosclerosis? Circulation. 2004;109(21 Suppl 1):II27-33.

20. Alp NJ, Mussa S, Khoo J, Cai S, Guzik T, Jefferson A, et al. Tetrahydrobiopterin-dependent preservation of nitric oxide-mediated endothelial function in diabetes by targeted transgenic GTPcyclohydrolase I overexpression. J Clin Invest. 2003;112(5):725-35.

21. Pacher P, Beckman JS, Liaudet L. Nitric oxide and peroxynitrite in health and disease. Physiol Rev. 2007;87(1):315-424.

22. Antonopoulos AS, Antoniades C, Tousoulis D, Bakogiannis C, Demosthenous M, Psarros C, et al. Novel therapeutic strategies targeting vascular redox in human atherosclerosis. Recent Pat Cardiovasc Drug Discov. 2009;4(2):76-87.

23. Frei B, Stocker R, Ames BN. Antioxidant defenses and lipid peroxidation in human blood plasma. Proceedings of the National Academy of Sciences of the United States of America. 1988;85(24):9748-52.

24. Tousoulis D, Kampoli AM, Papageorgiou N, Androulakis E, Antoniades C, Toutouzas K, et al. Pathophysiology of atherosclerosis: the role of inflammation. Current pharmaceutical design. 2011;17(37):4089-110.

25. Reilly SN, Jayaram R, Nahar K, Antoniades C, Verheule S, Channon KM, et al. Atrial sources of reactive oxygen species vary with the duration and substrate of atrial fibrillation: implications for the antiarrhythmic effect of statins. Circulation. 2011;124(10):1107-17.

26. Lee R, Margaritis M, Channon KM, Antoniades C. Evaluating oxidative stress in human cardiovascular disease: methodological aspects and considerations. Current medicinal chemistry. 2012;19(16):2504-20.

27. Schonbeck $U$, Libby P. Inflammation, immunity, and HMG-CoA reductase inhibitors: statins as antiinflammatory agents? Circulation. 2004;109(21 Suppl 1):II18-26.

28. Antonopoulos AS, Margaritis M, Shirodaria C, Antoniades C. Translating the effects of statins: from redox regulation to suppression of vascular wall inflammation. Thrombosis and haemostasis. 2012;108(5):840-8.

29. Sorrentino S, Landmesser U. Nonlipid-lowering effects of statins. Current treatment options in cardiovascular medicine. 2005;7(6):459-66. 
30. Koh KK, Sakuma I, Quon MJ. Differential metabolic effects of distinct statins. Atherosclerosis. 2011;215(1):1-8.

31. Sacks FM, Pfeffer MA, Moye LA, Rouleau JL, Rutherford JD, Cole TG, et al. The effect of pravastatin on coronary events after myocardial infarction in patients with average cholesterol levels. Cholesterol and Recurrent Events Trial investigators. The New England journal of medicine. 1996;335(14):1001-9.

32. Domanski M, Tian X, Fleg J, Coady S, Gosen C, Kirby R, et al. Pleiotropic effect of lovastatin, with and without cholestyramine, in the post coronary artery bypass graft (Post CABG) trial. The American journal of cardiology. 2008;102(8):1023-7.

33. Ridker PM, Danielson E, Fonseca FA, Genest J, Gotto AM, Jr., Kastelein JJ, et al. Rosuvastatin to prevent vascular events in men and women with elevated C-reactive protein. The New England journal of medicine. 2008;359(21):2195-207.

34. Brandes RP, Beer S, Ha T, Busse R. Withdrawal of cerivastatin induces monocyte chemoattractant protein 1 and tissue factor expression in cultured vascular smooth muscle cells. Arteriosclerosis, thrombosis, and vascular biology. 2003;23(10):1794-800.

35. Wei YM, Li X, Xiong J, Abais JM, Xia M, Boini KM, et al. Attenuation by Statins of Membrane Raft-Redox Signaling in Coronary Arterial Endothelium. J Pharmacol Exp Ther. 2013.

36. Wassmann S, Laufs U, Muller K, Konkol C, Ahlbory K, Baumer AT, et al. Cellular antioxidant effects of atorvastatin in vitro and in vivo. Arteriosclerosis, thrombosis, and vascular biology. 2002;22(2):300-5.

37. Inoue I, Goto S, Mizotani K, Awata T, Mastunaga T, Kawai S, et al. Lipophilic HMG-CoA reductase inhibitor has an anti-inflammatory effect: reduction of MRNA levels for interleukin-1beta, interleukin-6, cyclooxygenase-2, and p22phox by regulation of peroxisome proliferator-activated receptor alpha (PPARalpha) in primary endothelial cells. Life sciences. 2000;67(8):863-76.

38. Christ M, Bauersachs J, Liebetrau C, Heck M, Gunther A, Wehling M. Glucose increases endothelial-dependent superoxide formation in coronary arteries by $N A D(P) H$ oxidase activation: attenuation by the 3-hydroxy-3-methylglutaryl coenzyme A reductase inhibitor atorvastatin. Diabetes. 2002;51(8):2648-52.

39. Wagner AH, Kohler T, Ruckschloss U, Just I, Hecker M. Improvement of nitric oxidedependent vasodilatation by HMG-CoA reductase inhibitors through attenuation of endothelial superoxide anion formation. Arteriosclerosis, thrombosis, and vascular biology. 2000;20(1):61-9. 40. Mitani H, Egashira K, Ohashi N, Yoshikawa M, Niwa S, Nonomura K, et al. Preservation of endothelial function by the HMG-CoA reductase inhibitor fluvastatin through its lipid-lowering independent antioxidant properties in atherosclerotic rabbits. Pharmacology. 2003;68(3):121-30. 41. Tawfik HE, El-Remessy AB, Matragoon S, Ma G, Caldwell RB, Caldwell RW. Simvastatin improves diabetes-induced coronary endothelial dysfunction. J Pharmacol Exp Ther. 2006;319(1):386-95.

42. Wassmann S, Laufs U, Baumer AT, Muller K, Ahlbory K, Linz W, et al. HMG-CoA reductase inhibitors improve endothelial dysfunction in normocholesterolemic hypertension via reduced production of reactive oxygen species. Hypertension. 2001;37(6):1450-7.

43. Hong H, Zeng JS, Kreulen DL, Kaufman DI, Chen AF. Atorvastatin protects against cerebral infarction via inhibition of NADPH oxidase-derived superoxide in ischemic stroke. American journal of physiology Heart and circulatory physiology. 2006;291(5):H2210-5.

44. Vecchione C, Brandes RP. Withdrawal of 3-hydroxy-3-methylglutaryl coenzyme A reductase inhibitors elicits oxidative stress and induces endothelial dysfunction in mice. Circulation research. 2002;91(2):173-9.

45. Margaritis M, Channon KM, Antoniades C. Statins as regulators of redox state in the vascular endothelium: beyond lipid lowering. Antioxid Redox Signal. 2014;20(8):1198-215.

46. Laufs $U$, Liao JK. Post-transcriptional regulation of endothelial nitric oxide synthase mRNA stability by Rho GTPase. The Journal of biological chemistry. 1998;273(37):24266-71. 
47. Martinez-Gonzalez J, Raposo B, Rodriguez C, Badimon L. 3-hydroxy-3-methylglutaryl coenzyme a reductase inhibition prevents endothelial NO synthase downregulation by atherogenic levels of native LDLs: balance between transcriptional and posttranscriptional regulation.

Arteriosclerosis, thrombosis, and vascular biology. 2001;21(5):804-9.

48. Kosmidou I, Moore JP, Weber M, Searles CD. Statin treatment and 3' polyadenylation of eNOS mRNA. Arteriosclerosis, thrombosis, and vascular biology. 2007;27(12):2642-9.

49. Ota H, Eto M, Kano MR, Kahyo T, Setou M, Ogawa S, et al. Induction of endothelial nitric oxide synthase, SIRT1, and catalase by statins inhibits endothelial senescence through the Akt pathway. Arteriosclerosis, thrombosis, and vascular biology. 2010;30(11):2205-11.

50. de Sotomayor MA, Perez-Guerrero C, Herrrera MD, Jimenez L, Marin R, Marhuenda E, et al. Improvement of age-related endothelial dysfunction by simvastatin: effect on NO and COX pathways. British journal of pharmacology. 2005;146(8):1130-8.

51. Wilson SH, Simari RD, Best PJ, Peterson TE, Lerman LO, Aviram M, et al. Simvastatin preserves coronary endothelial function in hypercholesterolemia in the absence of lipid lowering. Arteriosclerosis, thrombosis, and vascular biology. 2001;21(1):122-8.

52. Aoki C, Nakano A, Tanaka S, Yanagi K, Ohta S, Jojima T, et al. Fluvastatin upregulates endothelial nitric oxide synthase activity via enhancement of its phosphorylation and expression and via an increase in tetrahydrobiopterin in vascular endothelial cells. Int J Cardiol. 2012;156(1):55-61.

53. Brouet A, Sonveaux P, Dessy C, Moniotte S, Balligand JL, Feron O. Hsp90 and caveolin are key targets for the proangiogenic nitric oxide-mediated effects of statins. Circulation research. 2001;89(10):866-73.

54. Ostad MA, Eggeling S, Tschentscher P, Schwedhelm E, Boger R, Wenzel P, et al. Flowmediated dilation in patients with coronary artery disease is enhanced by high dose atorvastatin compared to combined low dose atorvastatin and ezetimibe: results of the CEZAR study.

Atherosclerosis. 2009;205(1):227-32.

55. Rossoni LV, Wareing M, Wenceslau CF, Al-Abri M, Cobb C, Austin C. Acute simvastatin increases endothelial nitric oxide synthase phosphorylation via AMP-activated protein kinase and reduces contractility of isolated rat mesenteric resistance arteries. Clin Sci (Lond). 2011;121(10):44958.

56. Feron O, Dessy C, Desager JP, Balligand JL. Hydroxy-methylglutaryl-coenzyme A reductase inhibition promotes endothelial nitric oxide synthase activation through a decrease in caveolin abundance. Circulation. 2001;103(1):113-8.

57. Pelat M, Dessy C, Massion P, Desager JP, Feron O, Balligand JL. Rosuvastatin decreases caveolin-1 and improves nitric oxide-dependent heart rate and blood pressure variability in apolipoprotein E-/- mice in vivo. Circulation. 2003;107(19):2480-6.

58. Chen CA, Druhan LJ, Varadharaj S, Chen YR, Zweier JL. Phosphorylation of endothelial nitricoxide synthase regulates superoxide generation from the enzyme. The Journal of biological chemistry. 2008;283(40):27038-47.

59. Hattori Y, Nakanishi N, Akimoto K, Yoshida M, Kasai K. HMG-CoA reductase inhibitor increases GTP cyclohydrolase I mRNA and tetrahydrobiopterin in vascular endothelial cells. Arteriosclerosis, thrombosis, and vascular biology. 2003;23(2):176-82.

60. Wenzel P, Daiber A, Oelze M, Brandt M, Closs E, Xu J, et al. Mechanisms underlying recoupling of eNOS by HMG-CoA reductase inhibition in a rat model of streptozotocin-induced diabetes mellitus. Atherosclerosis. 2008;198(1):65-76.

61. Rueckschloss U, Galle J, Holtz J, Zerkowski HR, Morawietz H. Induction of NAD(P)H oxidase by oxidized low-density lipoprotein in human endothelial cells: antioxidative potential of hydroxymethylglutaryl coenzyme A reductase inhibitor therapy. Circulation. 2001;104(15):1767-72. 62. Pignatelli P, Carnevale R, Cangemi R, Loffredo L, Sanguigni V, Stefanutti C, et al. Atorvastatin inhibits gp91phox circulating levels in patients with hypercholesterolemia. Arteriosclerosis, thrombosis, and vascular biology. 2010;30(2):360-7. 
63. Morawietz H, Erbs S, Holtz J, Schubert A, Krekler M, Goettsch W, et al. Endothelial Protection, AT1 blockade and Cholesterol-Dependent Oxidative Stress: the EPAS trial. Circulation. 2006;114(1 Suppl):I296-301.

64. Antoniades C, Bakogiannis C, Tousoulis D, Reilly S, Zhang MH, Paschalis A, et al. Preoperative atorvastatin treatment in CABG patients rapidly improves vein graft redox state by inhibition of Rac1 and NADPH-oxidase activity. Circulation. 2010;122(11 Suppl):S66-73.

65. Antoniades C, Bakogiannis C, Leeson P, Guzik TJ, Zhang MH, Tousoulis D, et al. Rapid, direct effects of statin treatment on arterial redox state and nitric oxide bioavailability in human atherosclerosis via tetrahydrobiopterin-mediated endothelial nitric oxide synthase coupling. Circulation. 2011;124(3):335-45.

66. Zhou Q, Liao JK. Statins and cardiovascular diseases: from cholesterol lowering to pleiotropy. Current pharmaceutical design. 2009;15(5):467-78.

67. Maack C, Kartes T, Kilter H, Schafers HJ, Nickenig G, Bohm M, et al. Oxygen free radical release in human failing myocardium is associated with increased activity of rac1-GTPase and represents a target for statin treatment. Circulation. 2003;108(13):1567-74.

68. Elmadhun NY, Lassaletta AD, Chu LM, Liu Y, Feng J, Sellke FW. Atorvastatin increases oxidative stress and modulates angiogenesis in Ossabaw swine with the metabolic syndrome. The Journal of thoracic and cardiovascular surgery. 2012;144(6):1486-93.

69. Trochu JN, Mital S, Zhang X, Xu X, Ochoa M, Liao JK, et al. Preservation of NO production by statins in the treatment of heart failure. Cardiovascular research. 2003;60(2):250-8.

70. An LP, An SK, Wei XH, Fu SY, Wu HA. Atorvastatin improves cardiac function of rats with chronic cardiac failure via inhibiting Rac1/P47phox/P67phox-mediated ROS release. European review for medical and pharmacological sciences. 2015;19(20):3940-6.

71. Zheng Z, Jayaram R, Jiang L, Emberson J, Zhao Y, Li Q, et al. Perioperative Rosuvastatin in Cardiac Surgery. N Engl J Med. 2016;374(18):1744-53.

72. Kamath S, Blann AD, Lip GY. Platelet activation: assessment and quantification. European heart journal. 2001;22(17):1561-71.

73. Hartwich J, Dembinska-Kiec A, Gruca A, Motyka M, Partyka L, Skrzeczynska J, et al. Regulation of platelet adhesion by oxidized lipoproteins and oxidized phospholipids. Platelets. 2002;13(3):141-51.

74. Cha YJ, Chang EA, Kim CH. Effects of endothelial cells and mononuclear leukocytes on platelet aggregation. Haematologia. 2000;30(2):97-106.

75. . !!! INVALID CITATION !!!

76. Gaddam V, Li DY, Mehta JL. Anti-thrombotic effects of atorvastatin--an effect unrelated to lipid lowering. Journal of cardiovascular pharmacology and therapeutics. 2002;7(4):247-53.

77. Yemisci M, Ay H, Kocaefe C, Qui J, Topalkara K, Ozguc M, et al. Statin potentiates human platelet eNOS activity without enhancing eNOS mRNA and protein levels. Cerebrovascular diseases. 2008;26(2):190-8.

78. Puccetti L, Pasqui AL, Pastorelli M, Bova G, Cercignani M, Palazzuoli A, et al. Time-dependent effect of statins on platelet function in hypercholesterolaemia. European journal of clinical investigation. 2002;32(12):901-8.

79. Myung SK, Ju W, Cho B, Oh SW, Park SM, Koo BK, et al. Efficacy of vitamin and antioxidant supplements in prevention of cardiovascular disease: systematic review and meta-analysis of randomised controlled trials. BMJ. 2013;346:f10.

80. Dikalova AE, Bikineyeva AT, Budzyn K, Nazarewicz RR, McCann L, Lewis W, et al. Therapeutic targeting of mitochondrial superoxide in hypertension. Circulation research. 2010;107(1):106-16.

81. Maron BA, Michel T. Subcellular localization of oxidants and redox modulation of endothelial nitric oxide synthase. Circulation journal : official journal of the Japanese Circulation Society.

2012;76(11):2497-512.

82. Crea F, Niccoli G. Ezetimibe and Plaque Regression: Cholesterol Lowering or Pleiotropic Effects? Journal of the American College of Cardiology. 2015;66(5):508-10. 
83. Kalogirou M, Tsimihodimos V, Elisaf M. Pleiotropic effects of ezetimibe: do they really exist? European journal of pharmacology. 2010;633(1-3):62-70.

84. Tavori H, Rashid S, Fazio S. On the function and homeostasis of PCSK9: reciprocal interaction with LDLR and additional lipid effects. Atherosclerosis. 2015;238(2):264-70.

85. Ding Z, Liu S, Wang X, Deng X, Fan Y, Sun C, et al. Hemodynamic shear stress via ROS modulates PCSK9 expression in human vascular endothelial and smooth muscle cells and along the mouse aorta. Antioxid Redox Signal. 2015;22(9):760-71.

86. Sahebkar A, Di Giosia P, Stamerra CA, Grassi D, Pedone C, Ferretti G, et al. Effect of monoclonal antibodies to PCSK9 on high-sensitivity C-reactive protein levels: a meta-analysis of 16 randomized controlled treatment arms. British journal of clinical pharmacology. 2016;81(6):1175-90. 


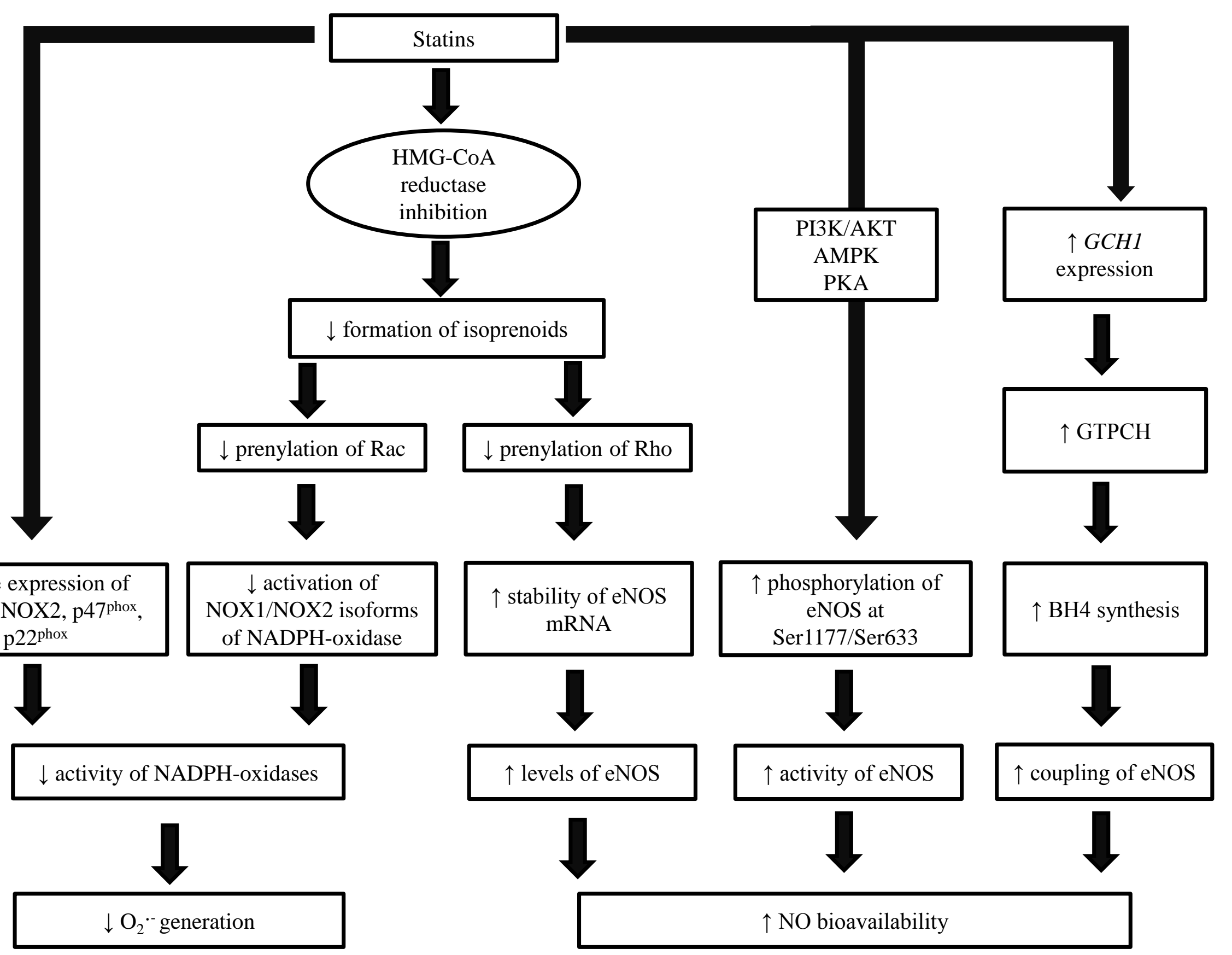

